\title{
Backpropagation-Based Cooperative Localization of Primary User for Avoiding Hidden-Node Problem in Cognitive Networks
}

\author{
Lin Liu, Zhengyi Li, and Chi Zhou \\ Department of Electrical and Computer Engineering, Illinois Institute of Technology, 3301 South Dearborn Street, \\ Chicago, IL 60616, USA \\ Correspondence should be addressed to Chi Zhou, zhouc@ece.iit.edu
}

Received 29 November 2009; Revised 24 March 2010; Accepted 28 April 2010

Academic Editor: Hsiao Hwa Chen

Copyright ( 92010 Lin Liu et al. This is an open access article distributed under the Creative Commons Attribution License, which permits unrestricted use, distribution, and reproduction in any medium, provided the original work is properly cited.

Cognitive radio (CR) is a technology to implement opportunistic spectrum sharing to improve the spectrum utilization. However, there exists a hidden-node problem, which can be a big challenge to solve especially when the primary receiver is passive listening. We aim to provide a solution to the hidden-node problem for passive-listening receiver based on cooperation of multiple CRs. Specifically, we consider a cooperative GPS-enabled cognitive network. Once the existence of PU is detected, a localization algorithm will be employed to first estimate the path loss model for the environment based on backpropagation method and then to locate the position of PU. Finally, a disable region is identified taking into account the communication range of both the PU and the CR. The CRs within the disabled region are prohibited to transmit in order to avoid interfering with the primary receiver. Both analysis and simulation results are provided.

\section{Introduction}

As more devices go wireless, spectrum becomes more and more crowded. Study of spectrum utilization, however, reveals that not all the spectrum is in use for all the time. Enforcement Bureau of Federal Communications Commission (FCC) measures the spectrum usage in Atlanta, Chicago, and so forth, and the study shows that only $5 \%-10 \%$ of the spectrum is used (up to $100 \mathrm{GHz}$ ) on the average. DARPA study reveals that only $2 \%$ of the allocated spectrum is used at any given time. Therefore, there is a potential to make efficient use of the unused spectrum without interfering with primary users (PUs) so that the spectrum utilization can be improved and more users can be supported. Cognitive radio (CR) is a technology to implement opportunistic spectrum sharing to improve the spectrum utilization [1-3]. CR can be applied in civilian applications, law enforcement, as well as military applications.

For CR, spectrum sensing is the first step but very crucial to the success. Only when the electromagnetic environment is thoroughly understood, it can be decided over which frequency to transmit and how to transmit. As the cognitive radio is seen as the secondary user to share the licensed band with the PU, they must avoid or control the interference to potential PU. However, as a radio device, a single CR may suffer severe shadowing or multipath fading with respect to primary transmitter so that it cannot detect the existence of primary transmitter even in its vicinities. In addition, there exists a hidden-node problem, in which a CR may be too far from the transmitter to detect the existence of the PU, but close to the primary receiver to interfere with the reception if transmit. Cooperative sensing provides a solution to the challenges mentioned above $[4,5]$. In cooperative sensing, multiple cognitive radios cooperate to reach an optimal global decision by exchanging and combining individual local sensing results. Allowing multiple CRs to cooperate, cooperative sensing can increase the detection probability, reduce the detection time, and achieve the diversity gain [611].

In this paper, we aim to provide a solution to the hidden-node problem for passive-listening receiver based on cooperation of multiple CRs. Solutions to hidden-node problem have been provided, such as RTS/CTS handshake for WLAN [12], BTMA (Busy Tone Multiple Access) for a centralized system [9], and DBTMA (Dual Busy Tone Multiple Access) for ad hoc networks [10]. However, most of 
the solutions require active participation of primary receiver and fail when primary receiver is just passive listening. When passive listening, primary receiver does not acknowledge or respond. In our approach, GPS-enabled CRs cooperate to first estimate the environment based on back propagation method and then locate the position of PU. Based on the PU location derived, we identify the disable region, whose radius is the sum of $\mathrm{PU}$ communication range and a CR communication range. Within the disable region, all the CRs are prohibited to transmit in order to avoid interfering with the PU. Since the disable region takes into account the CR communication range, our proposed method deals with the worst case of hidden-node problem. In addition, it does not require any involvement from primary receiver, suitable for passive-listening scenario.

The remainder of the paper is organized as follow. In Section 2, we present our system model. In Section 3, we propose the localization algorithm to first estimate the path loss model and further determine the exact location of PU. Simulation results are provided in Section 4, and finally the paper is concluded by Section 5 .

\section{System Model}

We consider a cognitive network, which consists of $N$ CRs that are GPS enabled and cooperative, shown in Figure 1. A simple energy detector is used by individual CR to detect the existence of PU by measuring the strength of the received power. A central control office collects the sensing results from individual $\mathrm{CR}$ to make a global detection decision. If the existence of PU is determined, the central control office runs a localization algorithm to first estimate the path loss model for the environment and then to locate the position of PU.

Once the position of PU is located, a disable region for CRs can be identified. Let $R_{p}$ denote the communication range for $\mathrm{PU}$ and $R_{c}$ denote the communication range for $\mathrm{CR}$, and then the disable region is the circle area with the origin at the PU location and the radius $R_{d}=R_{p}+R_{c}$. The actual CR communication range depends on the CR receiver and the CR transmits power jointly, which are known to the system. Therefore, it can be easily calculated given the path loss model. Finally, the central control office sends the control signal to individual CR to either disable or enable the CR depending on whether it locates within or outside of the disable region. The CRs within the disabled region are prohibited to transmit in order to avoid interfering with the primary receiver, while CRs outside of the region can transmit. Since the disable region takes into account the $\mathrm{CR}$ communication range, the hidden-node problem can be solved.

Widely adopted AODV (Ad Hoc On-Demand Distance Vector) routing protocol is used over a default clear channel to exchange the information between the CRs and the central control office. AODV protocol has small delay close to $8 \mathrm{~ms}$ in general and $20 \mathrm{k}$ to $30 \mathrm{k}$ routing overhead [13]. The default channel may be selected among several predetermined channels. A clear channel can be easily identified from the spectrum sensing stage.
In this paper, we use the log-normal shadowing path loss model:

$$
P_{r}(d)=P_{0}\left(d_{0}\right)+10 \cdot n \cdot \log \left(\frac{d_{0}}{d}\right)+X(0, \delta),
$$

where $P_{r}(d)$ is the received power at distance $d, P_{0}\left(d_{0}\right)$ is the received power at the reference distance $d_{0}, n$ is the path loss exponent, and $X(0, \delta)$ is normal shadowing random variable with zero mean and $\delta$ variance.

\section{Localization Algorithm}

In this section, we propose a localization algorithm, which will be employed to estimate the path loss model and further locate the position of PU once the presence of PU is detected. Since all CRs are GPS-enabled, the central control office can obtain the location information from those CRs. If we know the relative distances between PU and CRs, we can calculate the position of PU easily. As shown in (1), the distance between $\mathrm{PU}$ and a CR can be estimated by the received power for that CR and the path loss exponent $n$. Therefore, we need to estimate the path loss exponent first and then locate the PU.

\subsection{Localization Algorithm with Identical Path Loss Coeffi-} cients. We first consider the simple scenario in which all CRs locate in the same electromagnetic environment, that is, they have the same path loss exponent, expect for those CRs located close to PU, whose path loss should be modeled as free space path loss model. In (1), the reference point $d_{0}$ is always chosen in the close vicinity of PU; therefore, $P_{0}\left(d_{0}\right)$ can be calculated from free-space equation

$$
P_{0}\left(d_{0}\right)=\frac{P_{t} \cdot G_{t} \cdot G_{r} \cdot \lambda_{f}^{2}}{(4 \cdot \pi)^{2} \cdot d_{0}^{2}},
$$

where $P_{t}$ is the transmit power from the transmitter, $G_{t}$ and $G_{r}$ are the antenna gains for transmitter and receiver, respectively, and $\lambda_{f}$ is the wavelength of the carrier frequency.

Our localization algorithm is specified in details as follows (shown in Figure 2).

Step 1. Sort all CRs according to the descending order of the received power and number the CRs from 0 to $N-1$.

Step 2. If the received power of CR0 is above certain threshold, we pick CR0 as the reference, calculate the reference distance, and then continue the process. Otherwise, we will repeat Steps 1 and 2 till we find CR0 with received power exceeding the threshold.

That is, we treat the location of CR0 $\left(x_{0}, y_{0}\right)$ as the reference point and the received power of CR0 as $P_{0}\left(d_{0}\right)$. Then the reference distance $d_{0}$ can be calculated according to $(2)$ as

$$
d_{0}=\sqrt{\frac{P_{t} G_{t} \cdot G_{r} \cdot \lambda_{f}^{2}}{P_{0}\left(d_{0}\right)(4 \cdot \pi)^{2}}} .
$$




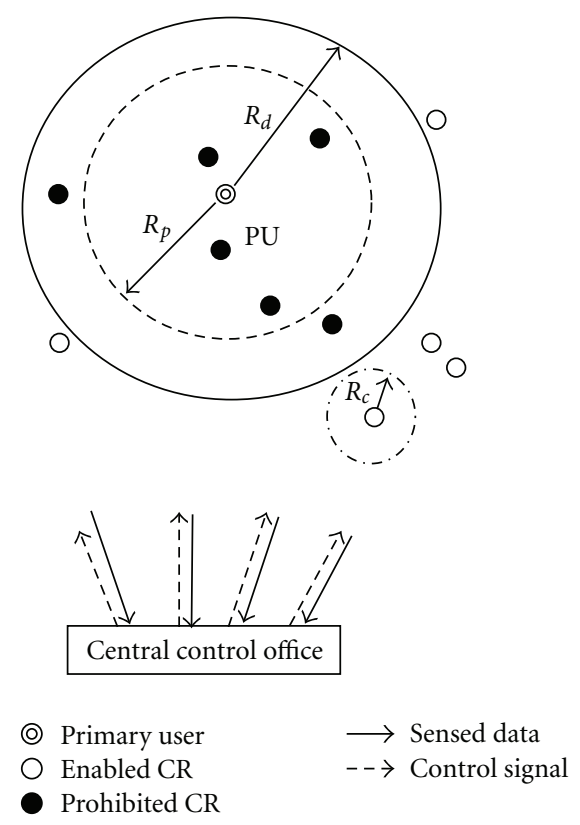

FIGURE 1: System model.

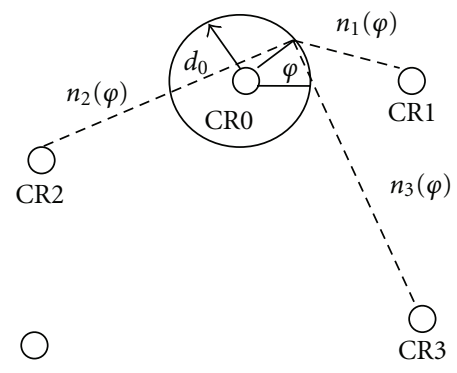

○

FIgURE 2: Illustration of Localization Algorithm.

From spectrum sensing stage, the carrier frequency of the PU can be identified. The transmit power is usually regulated to certain level. In the United States, FCC regulates the use of antennas not to exceed certain power limitations for wireless devices using the parameter EIRP (equivalent isotropically radiated power), which represents the effective transmit power of the radio in total, including transmit antenna gains. When using omnidirectional antennas (less than $6 \mathrm{~dB}$ ), the FCC rules require EIRP to be 1 watt or less. In this paper, we use $P_{t} G_{t}=1$ watt.

The choice of the power threshold depends on how much accuracy or estimation error the system can tolerate. One example is illustrated in Figure 4.

Step 3. Draw a circle with the CR0 location as the center and $d_{0}$ as the radius. If CR0 does follow the free-space propagation, the PU must locate on that circle.
Step 4. Pick the next three CRs, CR1, CR2, and CR3, in the list. Move the PU location along the circle from 0-degree angle to 360-degree angle and calculate the corresponding path loss exponent $n$ for CR1, CR2, and CR3, respectively.

For example, we consider CR1. When the PU locates on the circle with angle $\varphi$, the PU location is $\left(x_{0}+d_{0} \cos \varphi, y_{0}+\right.$ $d_{0} \sin \varphi$ ), and the distance between the point and CR1 is

$$
d_{1}=\sqrt{\left(x_{0}+d_{0} \cos \varphi-x_{1}\right)^{2}+\left(y_{0}+d_{0} \sin \varphi-y_{1}\right)^{2}} .
$$

According to (1), when ignoring the shadowing, the path loss exponent is

$$
n_{1}(\varphi)=\frac{P_{0}\left(d_{0}\right)-P_{1}}{10 \log \left(d_{1} / d_{0}\right)}
$$

Step 5. Calculate the relative difference between the path loss exponents among those three CRs for any angle $\varphi$ :

$$
\begin{aligned}
& E 12=\left|n_{1}(\varphi)-n_{2}(\varphi)\right|, \\
& E 13=\left|n_{1}(\varphi)-n_{3}(\varphi)\right|, \\
& E 32=\left|n_{3}(\varphi)-n_{2}(\varphi)\right| .
\end{aligned}
$$

We pick the angle that gives the minimum difference summation:

$$
\bar{\varphi}=\arg \min (E 12+E 13+E 32) .
$$

Then the path loss exponent $\bar{n}$ can be estimated as

$$
\bar{n}=\frac{n_{1}(\bar{\varphi})+n_{2}(\bar{\varphi})+n_{3}(\bar{\varphi})}{3} .
$$

The position of PU can be estimated as

$$
\begin{aligned}
& \hat{x}=x_{0}+d_{0} \cos \bar{\varphi} \\
& \hat{y}=y_{0}+d_{0} \sin \bar{\varphi} .
\end{aligned}
$$

Let $P_{\min }$ be the minimum acceptable received signal power for the main receiver from PU. Ignoring the random shadowing, we can calculate the communication range of $\mathrm{PU}$ as

$$
R_{p}=\frac{10^{\left(P_{0}\left(d_{0}\right)-P_{\min }\right) / 10 \bar{n}}}{d_{0}} .
$$

And then the radius for disable region for CRs is

$$
R_{d}=R_{p}+R_{c} .
$$

Without shadowing, our algorithm can accurately estimate the path loss exponent and locate the exact location of PU. When there is shadowing, the shadowing effect can be reduced if we take multiple samples of the received power to average out the randomness. 
CR0, though having the strongest received power, may not follow the free-space path loss model. In this case, we need to evaluate the proposed localization algorithm in terms of error, which is defined as

$$
\text { Error }=\frac{d_{e}}{R_{p}}=\frac{\sqrt{\left(x_{\mathrm{PU}}-\hat{x}\right)^{2}-\left(y_{\mathrm{PU}}-\hat{y}\right)^{2}}}{R_{p}} \text {, }
$$

where $d_{e}$ is the separation distance between the actual PU and the estimated PU, $\left(x_{\mathrm{PU}}, y_{\mathrm{PU}}\right)$ is the actual PU location, and $(\hat{x}, \hat{y})$ is the estimated PU location. Some simulation results will be given in Section 4 .

\subsection{Localization Algorithm with Variant Path Loss Coefficients.} It is likely that the CRs locate in different environment; thus, we propose a backpropagation-based algorithm to locate the PU under a variant environment. Back propagation has been used in various areas, such as the artificial neural network and the MIMO process, but no work has been found in communication networks to the best of our knowledge.

As the algorithm's name implies, the errors (and therefore the learning) propagate backwards from the output nodes to the inner nodes in a network. So technically speaking, back propagation is used to calculate the gradient of the error of the network with respect to the network's modifiable weights. This gradient is always then used in a simple stochastic gradient descent algorithm to find weights that minimize the error. Often the term "back propagation", used in a more general sense, refers to the entire procedure encompassing both the calculation of the gradient and its use in stochastic gradient descent. Back propagation usually allows quick convergence on satisfactory local minima for error in some appropriate network settings.

In this paper, we can consider our model as a 2-input/1output system. All we have to do is to estimate all the weights and hidden nodes in the system to approximate the true results. According to (1), there are two unknown variables in the equation: $d$ and $n$. In the variant environment, path loss coefficients can be varied. But we can first estimate the rough position of PU using AOA estimation method. This result will not provide us an acceptable result. However, it can eliminate one variable in (1). In this way, we can employ the back propagation algorithm to find out the path loss coefficients of all the cognitive radios. Using the results of back propagation algorithm, we can further estimate the exact location of the PU.

For back propagation algorithm, we can treat (1) in the form as below:

$$
P_{r}=f(n, d)
$$

The received power calculated from (13) varies from the true value of the received power depending on the values of the path loss coefficients and the estimated distances. As we talk above, we can find a way to first estimate the location of the PU with a larger error. In order to get the closest result to the true value, we introduce (14) to calculate the square error.
Finding a good estimation of the path loss coefficients will now become finding the minimum value of function $J(n)$ :

$$
J(n)=\left(f(n, d)-P_{\text {real }}\right)^{2},
$$

where $P_{\text {real }}$ is the actual measured received power.

We start finding the proper path loss coefficients with a set of random initial values. Then we process those variables with the back propagation algorithm. The algorithm is described as shown in the following steps

Step 1 and Step 2. are the same as those in localization algorithm with the identical path loss.

Step 3. CR0 estimates the angle of arrival (AOA), $\theta$, of the received signal. The rough position of the PU can be calculated as

$$
\begin{aligned}
& \hat{y}_{\text {Initial }}=y_{0}+d_{0} \sin \theta, \\
& \hat{x}_{\text {Initial }}=x_{0}+d_{0} \cos \theta .
\end{aligned}
$$

Step 4. Using the back propagation algorithm [14] to obtain the path loss coefficients for every cognitive radio. The detail process can be described as follows

(1) Pick initial values of the path loss coefficient for each CR using pseudorandom process that randomly selects $n_{i}$ within the range from 2 to 8 for the $i$ th CR.

(2) Compute the receive power of each CR with the coefficients according to (13).

(3) Compute the objective function

$$
J\left(n_{1}, \ldots, n_{M}\right)=\frac{1}{M} \sum_{i=1}^{M}\left(f\left(n_{i}, d_{i}\right)-P_{i, \text { real }}\right)^{2},
$$

where $M$ is the number of the CRs that we use in the algorithm, $f\left(n_{i}, d_{i}\right)$ is the estimated received power calculated using the selected $n_{i}$ for the $i$ th CR, and $P_{i \text {,real }}$ is the actual measured received power obtained by the antenna of the $i$ th $\mathrm{CR}, n_{1}$ to $n_{M}$ is the path loss exponent that we need to obtain in this step.

(4) Take the second-order derivative of $J\left(n_{1}, n_{2}, \ldots, n_{M}\right)$ with respect to each path loss coefficient $n_{i}$ and select the smallest value as the updating step $\eta$ :

$$
\begin{gathered}
\eta=\min \left(\frac{\partial J^{2}\left(n_{1}, \ldots, n_{M}\right)}{\partial n_{1}^{2}}, \frac{\partial J^{2}\left(n_{1}, \ldots, n_{M}\right)}{\partial n_{2}^{2}}, \ldots,\right. \\
\left.\frac{\partial J^{2}\left(n_{1}, \ldots, n_{M}\right)}{\partial n_{M}^{2}}\right) .
\end{gathered}
$$

(5) Update the parameters $n_{i}$ using the following equation:

$$
n_{i}^{L+1}=n_{i}^{L}+\eta \frac{\partial J\left(n_{1}, \ldots, n_{M}\right)}{\partial n_{i}},
$$

where $L$ denotes the iteration number.

(6) Repeat procedures (2) to (5) until we have a decent estimation. Usually we have an acceptable answer after 70 iterations. 
Step 5. Compute distance between the PU and every CR using the equation given below:

$$
d_{i}=\frac{d_{0}}{10^{\left(P_{i, \text { real }}-P_{o}\left(d_{o}\right)\right) / 10 \hat{n}_{i}}} .
$$

Step 6. Determine the area that the PU might locate, which is specified by the intersections of each circle centered at each CR with the estimated $d_{i}$ from Step 5 as the radius.

Step 7. Compute a 3D probability contour and pick the point giving us the largest probability as the PU location. The calculation of the probability for point $c$ on the intersection plane is given as (20):

$$
\text { Prob }=\prod \operatorname{pdf}\left\{N\left(P_{i, \text { real }}-P d_{0}-10 \hat{n}_{i} \cdot \log \left(\frac{d_{0}}{d_{c, i}}\right), \delta\right)\right\}
$$

where $d_{c, i}$ is the distance between point $c$ and the $i$ th CR.

\section{Simulation Results}

4.1. Localization Simulation with Identical Environment. We consider a cooperative GPS-enabled cognitive network with the following parameter setup: PU location: $(22,23)$, CR0 location: $(22,22)$, CR1 location: $(6,5)$, CR2 location: (40, 6), CR3 location: $(7,41), P_{t} G_{t}=1 \mathrm{watt}, G_{r}=1$, and carrier frequency is $300 \mathrm{MHz}$. In all simulations, we choose $-4.5 \mathrm{dbm}$ as the power threshold (illustrated in Figure 4).

We further assume that the path loss for CR0 follows freespace equation, and CR1, CR2, and CR3 have the same path loss exponent, $n=4$. We consider two scenarios, without shadowing and with shadowing.

4.1.1. Without Shadowing. In this case, the path loss model is simplified to log-distance model. This is a deterministic model without randomness. We plot the corresponding path loss exponent estimate for CR1, CR2, and CR3, respectively, as we move the potential $\mathrm{PU}$ point along the circle from 0 degree to 360 degrees.

From Figure 3, we can see that the path loss exponent varies as the point moves, as the distance between the potential PU and CR changes. In addition, the three curves cross at a single point with the angle $\varphi=90$ degrees and the corresponding path loss exponent $n=4$. According to (6) to (8), the crossing point has the minimum summed differences; therefore, we have $\bar{\varphi}=90$ degrees and path loss exponent $\bar{n}=4$. That is, the estimations perfectly match the exact values.

We then examine how to choose the power threshold in step 2 to determine whether a CR can be considered as the reference. We assume that the reference distance is $d_{0}=1$ and the actual path loss for the CR is 4 . In general, the path loss exponent varies between 2 and 4 . Therefore, we consider the worst case. We vary the distance between the PU and the CR and calculate the corresponding error using (12). The results are illustrated in Figure 4. It is shown that the error increases with the distance. If we choose the $5 \%$ error, the distance is 2 and the corresponding received power is $-4.5 \mathrm{dbm}$, which is the chosen power threshold.
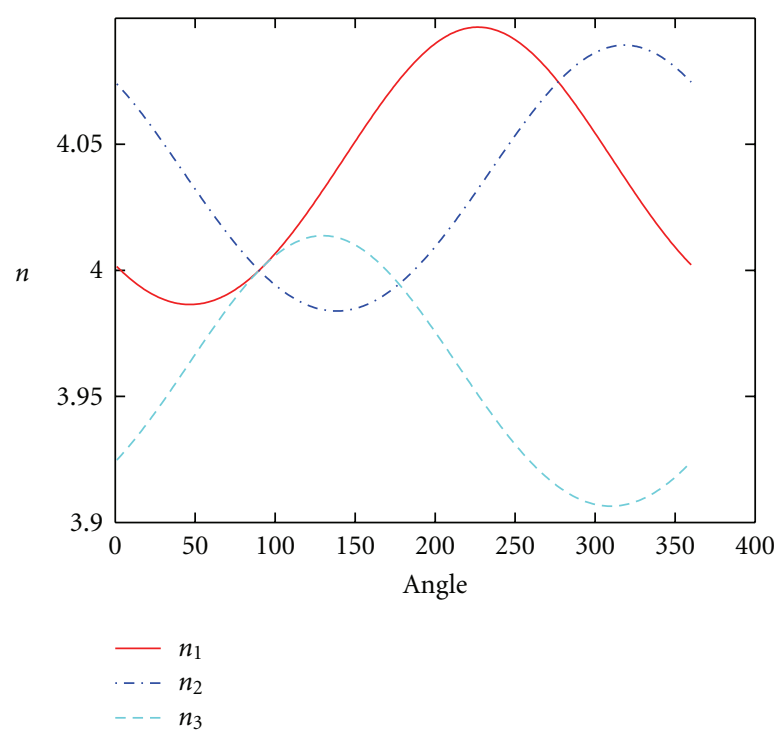

FIgURE 3: Path loss exponent versus angle without shadowing.

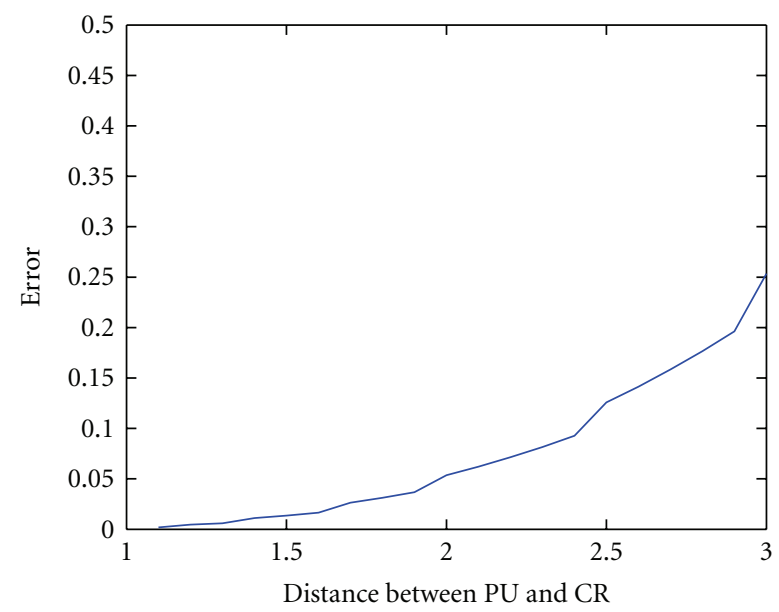

Figure 4: Error versus the distance between the PU and CR0.

4.1.2. With Shadowing. Then we add random shadowing into the path loss model. We first set the shadowing random variable with zero mean and $8 \mathrm{~dB}$ variance. To average out the randomness, we take 600 samples and average the received power, $P_{1}, P_{2}$, and $P_{3}$. We redraw the path loss exponent curves as we move the potential PU point along the circle from 0 degree to 360 degrees, shown in Figure 5. The three curves do not intersect at a single point in contrast to the noshadowing case. Again, we use (6) to (8) to estimate the angle $\bar{\varphi}$ and path loss exponent $\bar{n}$. It is shown that at 99 degrees the three exponents have the minimum summed difference, that is, $\bar{\varphi}=99$ degrees and $\bar{n}=4.05$. Then the estimated location for PU is $(21.8491,22.9529)$. As we can see, the estimated position is very close to the exact location of PU.

We would like to see how the increase of the sample number would affect the performance. Therefore, we increase the number of samples, and check the estimated location of PU. Figure 6 shows how the estimated $x$-coordinate varies 


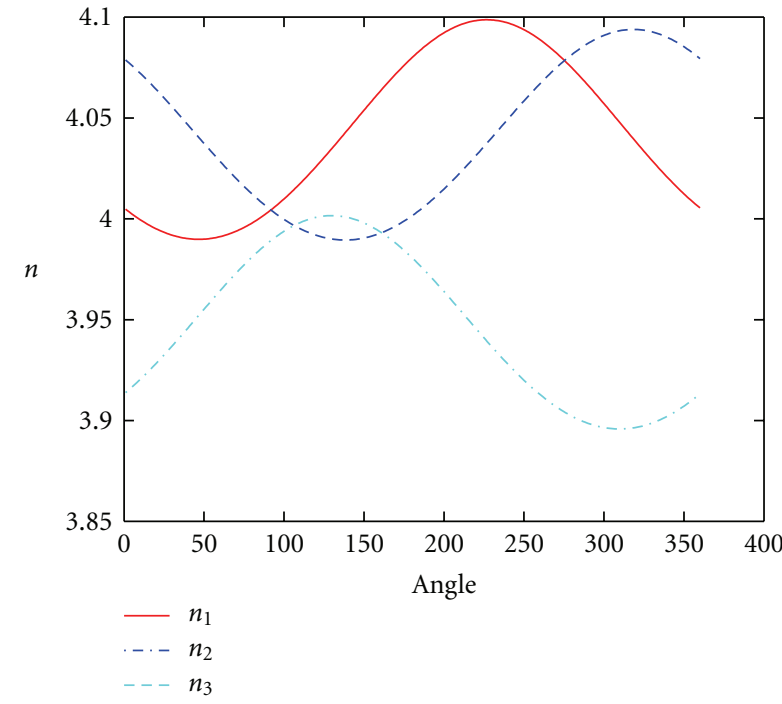

FIGURe 5: Path loss exponent versus angle using 600 samples with shadowing.

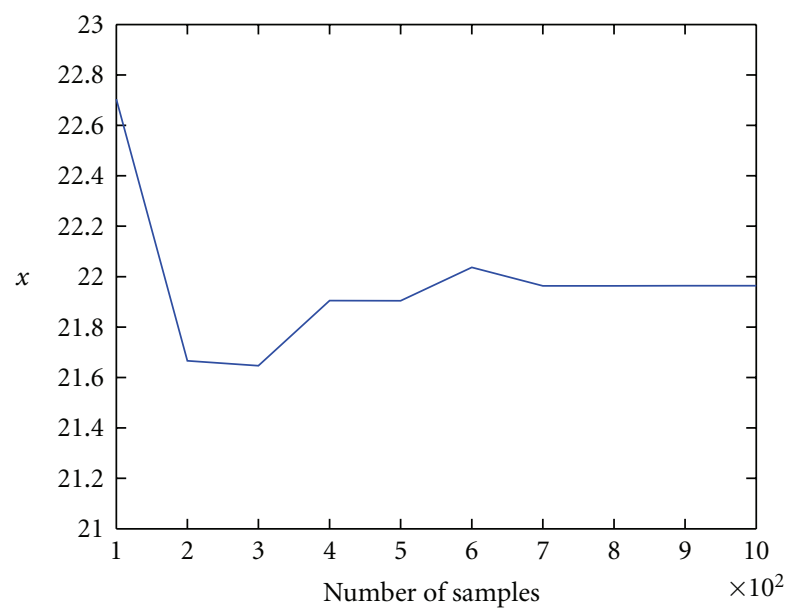

FIGURE 6: $x$ coordinate of PU location versus sample number.

TABLe 1

\begin{tabular}{lccccc}
\hline Sample number & 100 & 200 & 300 & 400 & 500 \\
\hline Angle & 65 & 134 & 77 & 63 & 71 \\
Sample number & 600 & 700 & 800 & 900 & 1000 \\
Angle & 80 & 92 & 92 & 92 & 92 \\
\hline
\end{tabular}

with the number of samples. It is shown that the estimated value approaches to the exact value as the number of samples increases. In this specific example, we observe that the curve reaches the maximum error at 200 samples; however, the difference is still less than 1 , which is less than $5 \%$ as an error. In addition, the curve approaches to the exact 22 as the number of samples increases. The same property can be found for $y$ coordinate.

We also estimate the angle given the various number of samples, as shown in Table 1 .

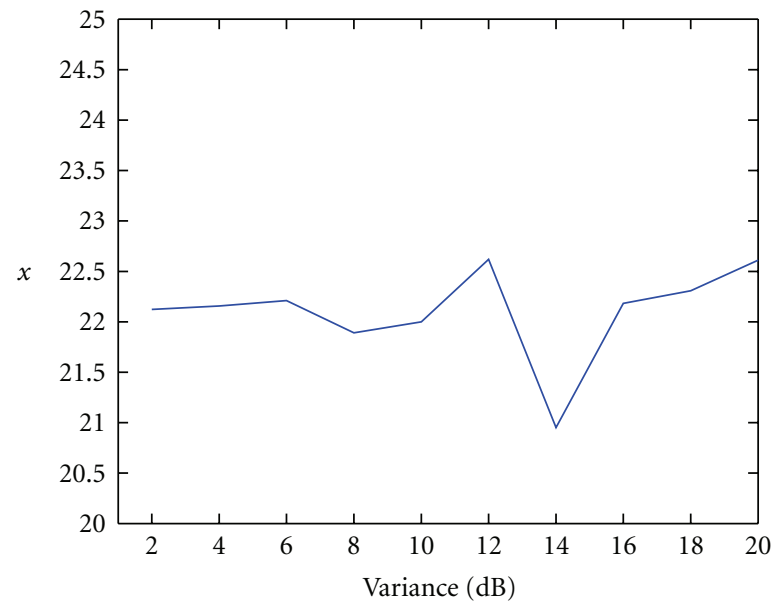

FIGURE 7: $x$-coordinate of PU location versus variance of shadowing.

As we can observe here, the estimated angle also approaches to the exact value 90 of degrees as the number of samples increases. When more than 800 samples are used, we can get the estimated angle really close to the exact value of 90 degree with only 1 or 2 degrees difference.

In addition, we want to study the effect of the shadowing variance casting on the accuracy of the localization. In this simulation, we use 1000 samples for each outcome under different variance.

Figure 7 shows the estimated $x$-coordinate for different shadowing variance. We can see that the difference between the exact value and estimated value tends to become larger as we increase the variance of shadowing. If we choose the variance less than $10 \mathrm{~dB}$, we can estimate the position of PU within small error using the proposed localization algorithm. Similar properties are found for $y$-coordinate and the estimated angle.

4.2. Localization Simulation with Variant Environment. In this section, we focus on the performance of the localization algorithm with variant environment. We assume that $\mathrm{PU}$ location $(23,23)$ and path losses for CR1, CR2, and CR3 are 5,4 , and 6 , respectively, while keeping other parameters unchanged as $A$ with the shadowing random variable with zero mean and $8 \mathrm{~dB}$ variance.

The back propagation algorithm is applied to estimate the path loss coefficients. Figure 8 shows the estimated coefficients versus the number of iterations. It is shown that the back propagation algorithm takes approximately 100 iterations to reach the steady final result. Note that the number of iteration needed varies with the distance between the PU and the reference point.

Once the path loss coefficients are estimated, we process Step 6 to determine in which area the PU may locate. We calculate the estimated distance between the PU and each cognitive radio and then draw a circle which indicates the probable area of the PU. Then we take all the intersections and overlaps into consideration and identify the area for PU. 


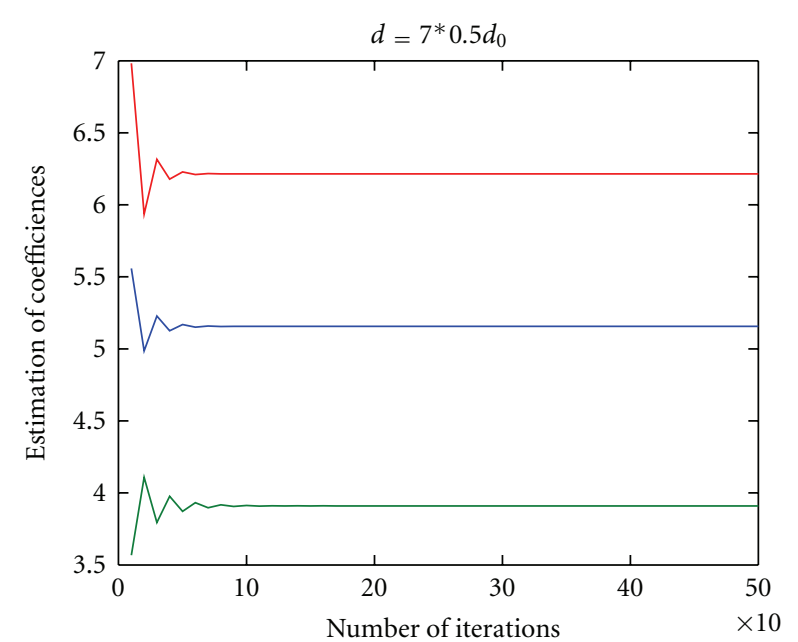

$-n_{1}$

$-n_{2}$

FIGURE 8: Estimation of path loss coefficients versus iterations number.

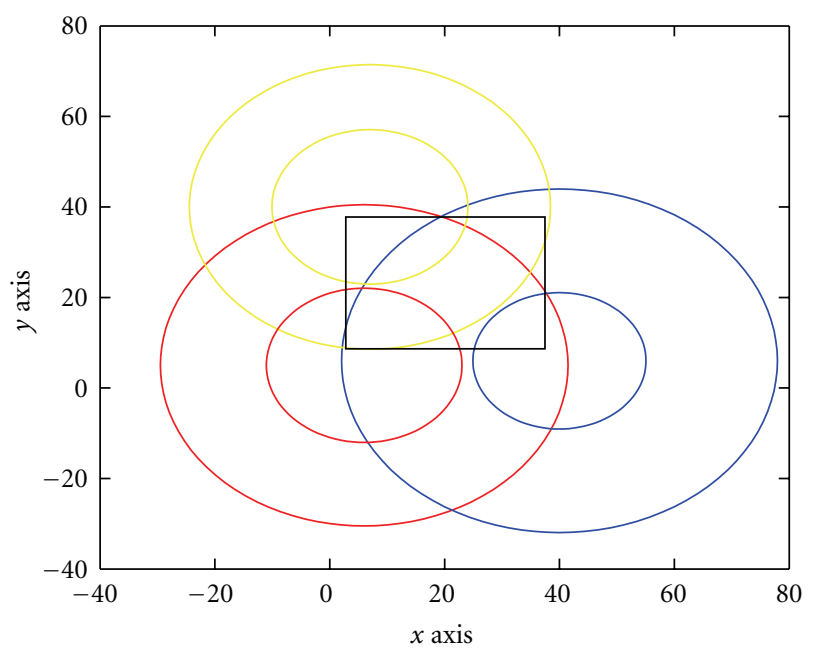

FIGURE 9: Probable area for the location of PU.

The result is given in Figure 9, marked as a gray rectangle. Compute the probability for each point in the black rectangle according to (20) and plot the 3-D probability contour in Figure 10. The corresponding 2D contour mapping is shown in Figure 11. From Figures 10 and 11, we chose the point with the largest possibility as the estimated PU location, that is, $(22.4702,22.9886)$. This estimation is really close to the actual location $(23,23)$.

The key of the algorithm is to get the precise estimation of the path loss coefficients following Step 4, as those estimations will be used to determine the PU location. The estimation accuracy depends on the reference point. The path loss for the reference point has been assumed to follow free-space propagation. Figure 12 shows how the actual path loss for the reference point affects the accuracy. It is shown

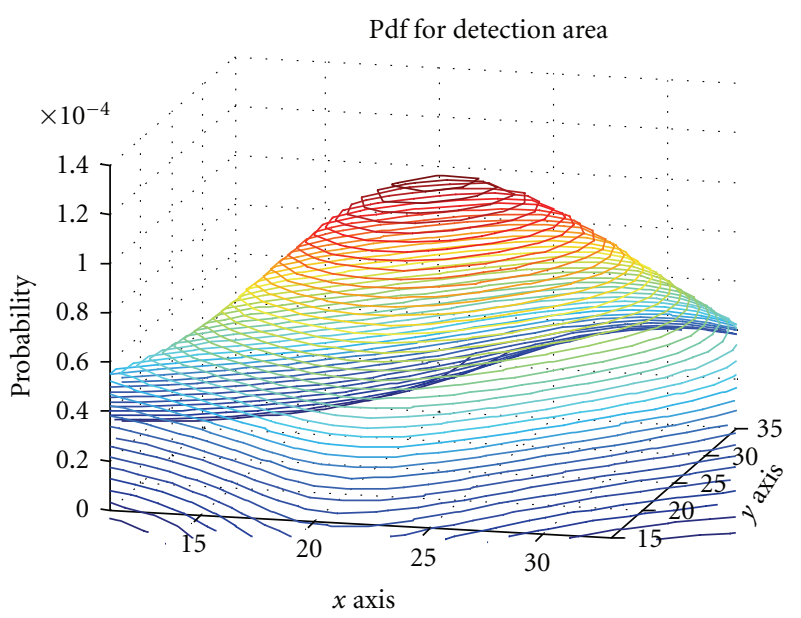

Figure 10: 3-D probability contour of the detection area.

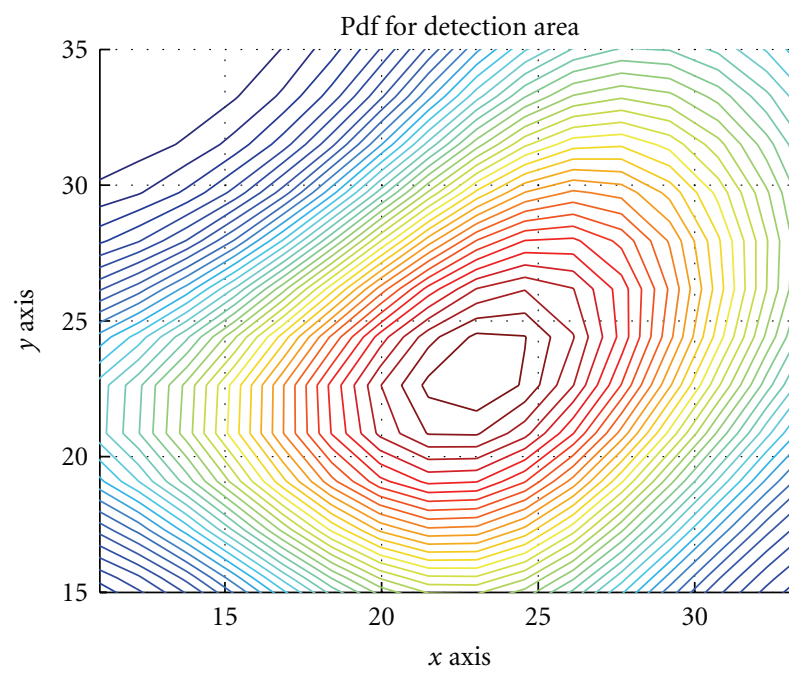

FIgURE 11: Corresponding 2-D contour mapping.

that the effect is not that significant. We also check how the distance between the reference point and the PU location affects the accuracy, shown in Figure 13. It is shown that as the reference point is further away from the PU, the estimation gets worse. Consequently, the localization error follows the same trend, shown in Figure 14. The localization error increases with the reference distance. Thus, in order to achieve desired accuracy, the reference point should be close enough to the PU. For this specific example, the reference node should be chosen within 1.2 units away from the PU for good accuracy.

\section{Conclusion}

In this paper, we have proposed a solution to the hiddennode problem in a cooperative GPS enabled cognitive network. Our proposed localization algorithm estimates 


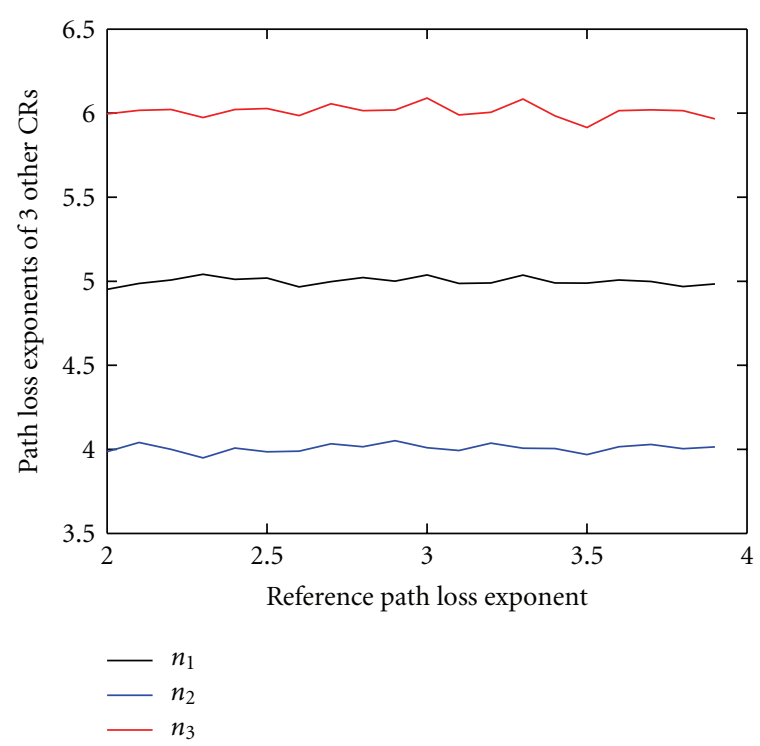

Figure 12: Estimated path loss of 3 CRs versus the actual path loss of reference point.

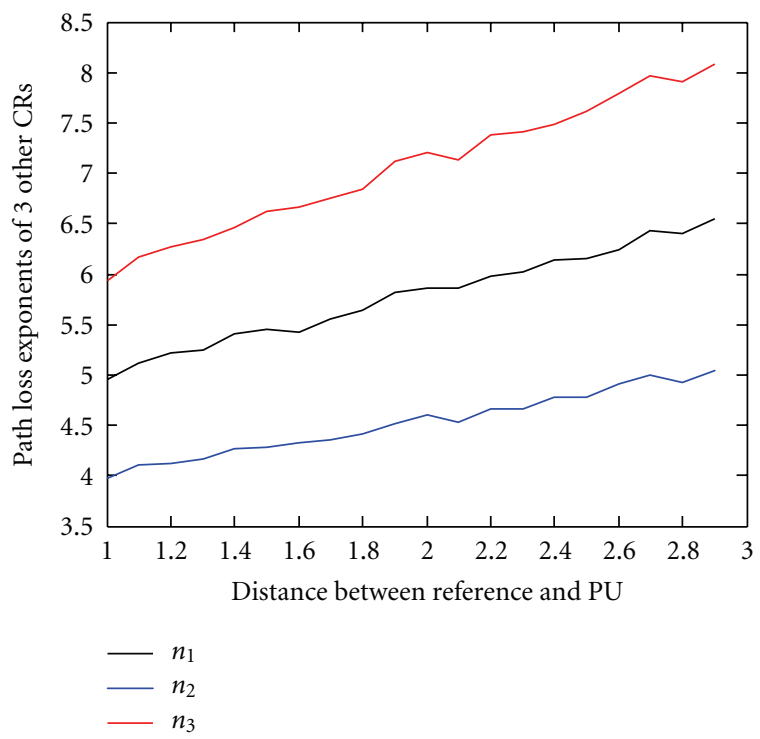

FIGURE 13: Estimated path loss of 3 CRs versus the distance between reference point and the PU location.

the path loss model and then locates the position of PU. Consequently, we can determine the disable region for CRs to avoid the interference to PU as well as the hiddennode problem. The simulation results have shown that the localization algorithm can provide accurate location results given that the reference node is close enough.

\section{Acknowledgments}

This work was supported by AFOSR under Grant no. FA9550-09-0630 and by NSF under Grant no. CNS 0619693.

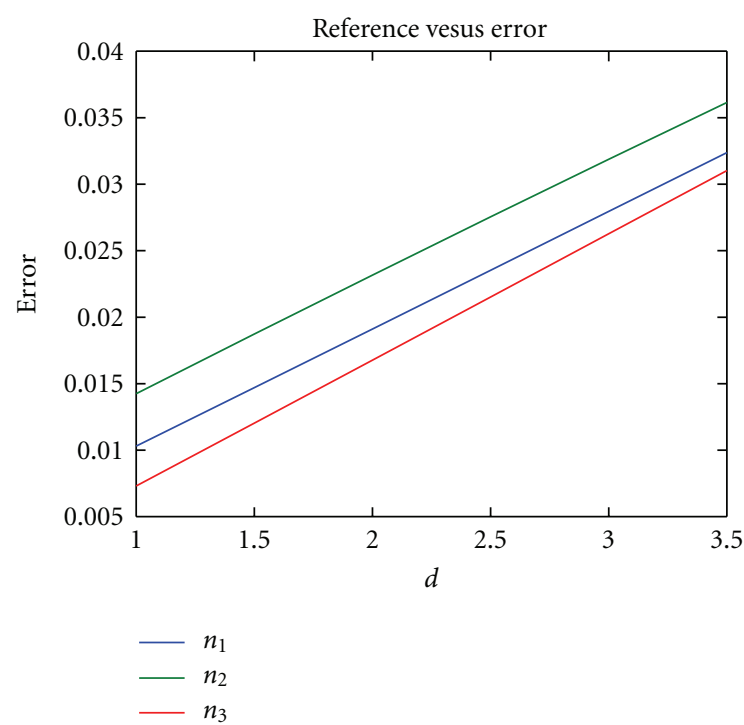

FIGURE 14: Algorithm error versus reference location.

\section{References}

[1] J. Mitola, Cognitive radio, licentiate thesis, KTH, Stsockholm, Sweden, 1999.

[2] S. Haykin, "Cognitive radio: brain-empowered wireless communications," IEEE Journal on Selected Areas in Communications, vol. 23, no. 2, pp. 201-220, 2005.

[3] “Report FCC 05-57," ET Docket no. 03-108, March 2005.

[4] A. Ghasemi and E. S. Sousa, "Collaborative spectrum sensing for opportunistic access in fading environments," in Proceedings of the 2005 1st IEEE International Symposium on New Frontiers in Dynamic Spectrum Access Networks (DySPAN '05), pp. 131-136, Baltimore, Md, USA, November 2005.

[5] G. Ganesan and Y. Li, "Cooperative spectrum sensing in cognitive radio networks," in Proceedings of the 1st IEEE International Symposium on New Frontiers in Dynamic Spectrum Access Networks (DySPAN '05), pp. 137-143, Baltimore, Md, USA, November 2005.

[6] G. Ganesan and Y. Li, "Cooperative spectrum sensing in cognitive radio, part II: multiuser networks," IEEE Transactions on Wireless Communications, vol. 6, no. 6, pp. 2214-2222, 2007.

[7] G. Ganesan and Y. Li, "Cooperative spectrum sensing in cognitive radio, part I: two user networks," IEEE Transactions on Wireless Communications, vol. 6, no. 6, pp. 2204-2212, 2007.

[8] J. N. Laneman, D. N. C. Tse, and G. W. Wornell, "Cooperative diversity in wireless networks: efficient protocols and outage behavior," IEEE Transactions on Information Theory, vol. 50, no. 12, pp. 3062-3080, 2004.

[9] F. A. Tobagi and L. Kleinrock, "Packet switching in radio channels: part II-the hidden terminal problem in carrier sense multiple-access and the busy-tone solution," IEEE Transactions on Communications, vol. 23 , no. 12, pp. 14171433, 1975.

[10] Z. J. Haas and J. Deng, "Dual busy tone multiple access (DBTMA)-a multiple access control scheme for ad hoc networks," IEEE Transactions on Communications, vol. 50, no. 6, pp. 975-985, 2002. 
[11] G. Ganesan and Y. Li, "Agility improvement through cooperative diversity in cognitive radio," in Proceedings of the IEEE Global Telecommunications Conference (GLOBECOM '05), pp. 2505-2509, St. Louis, MO, usa, December 2005.

[12] "Wireless LAN Medium Access Control (MAC) and Physical Layer (PHY) Specifications," ANSI/IEEE Std 802.11: 1999 (E) Part 11, ISO/IEC 880211, 1999.

[13] A. H. A. Rahman and Z. A. Zukarnain, "Performance comparison of AODV, DSDV and I-DSDV routing protocols in mobile ad hoc networks," European Journal of Scientific Research, vol. 31, no. 4, pp. 566-576, 2009.

[14] R. O. Duda, P. E. Hart, and D. G. Stork, Pattern Classification, John Wiley \& Sons, New York, NY, USA, 1973. 

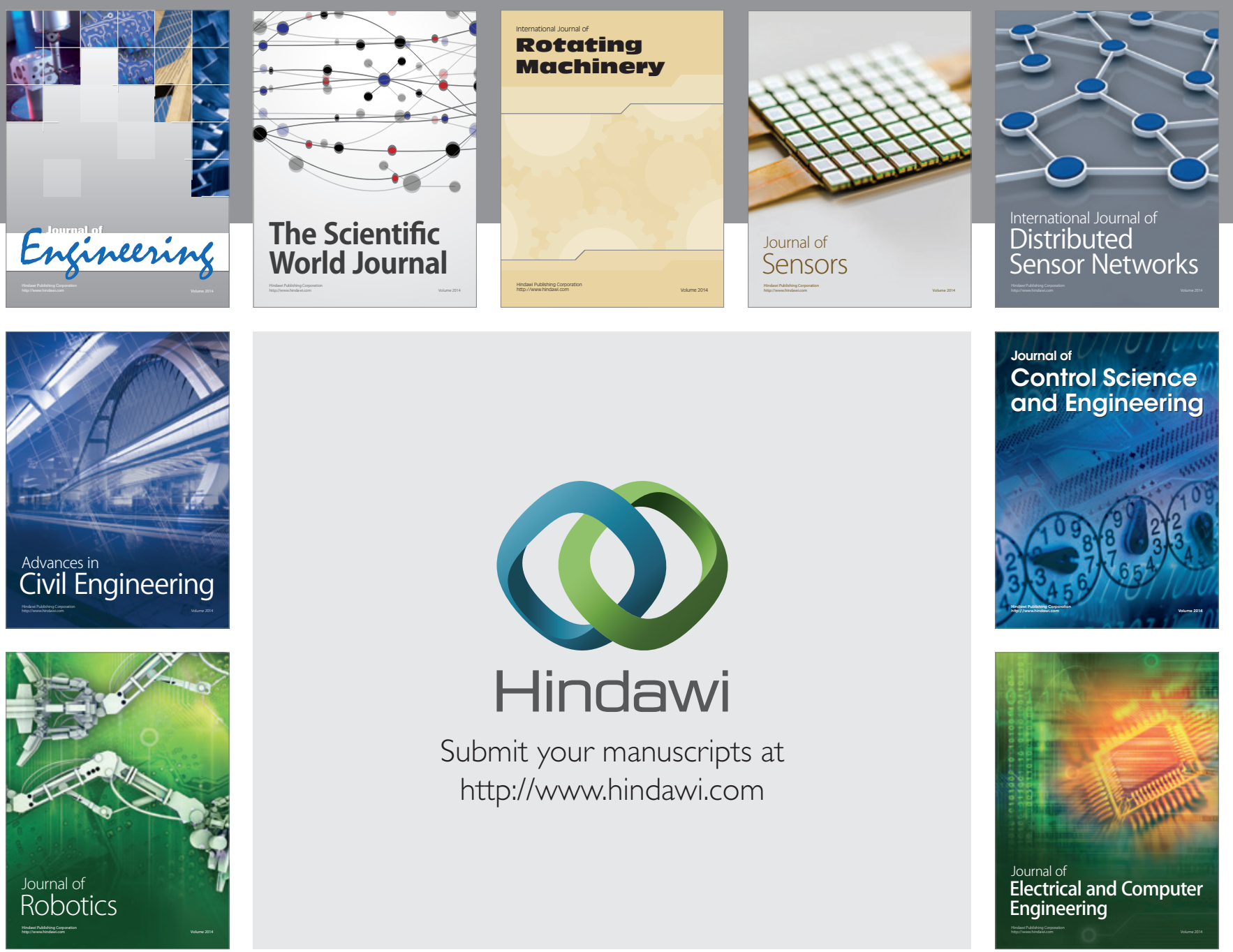

Submit your manuscripts at

http://www.hindawi.com
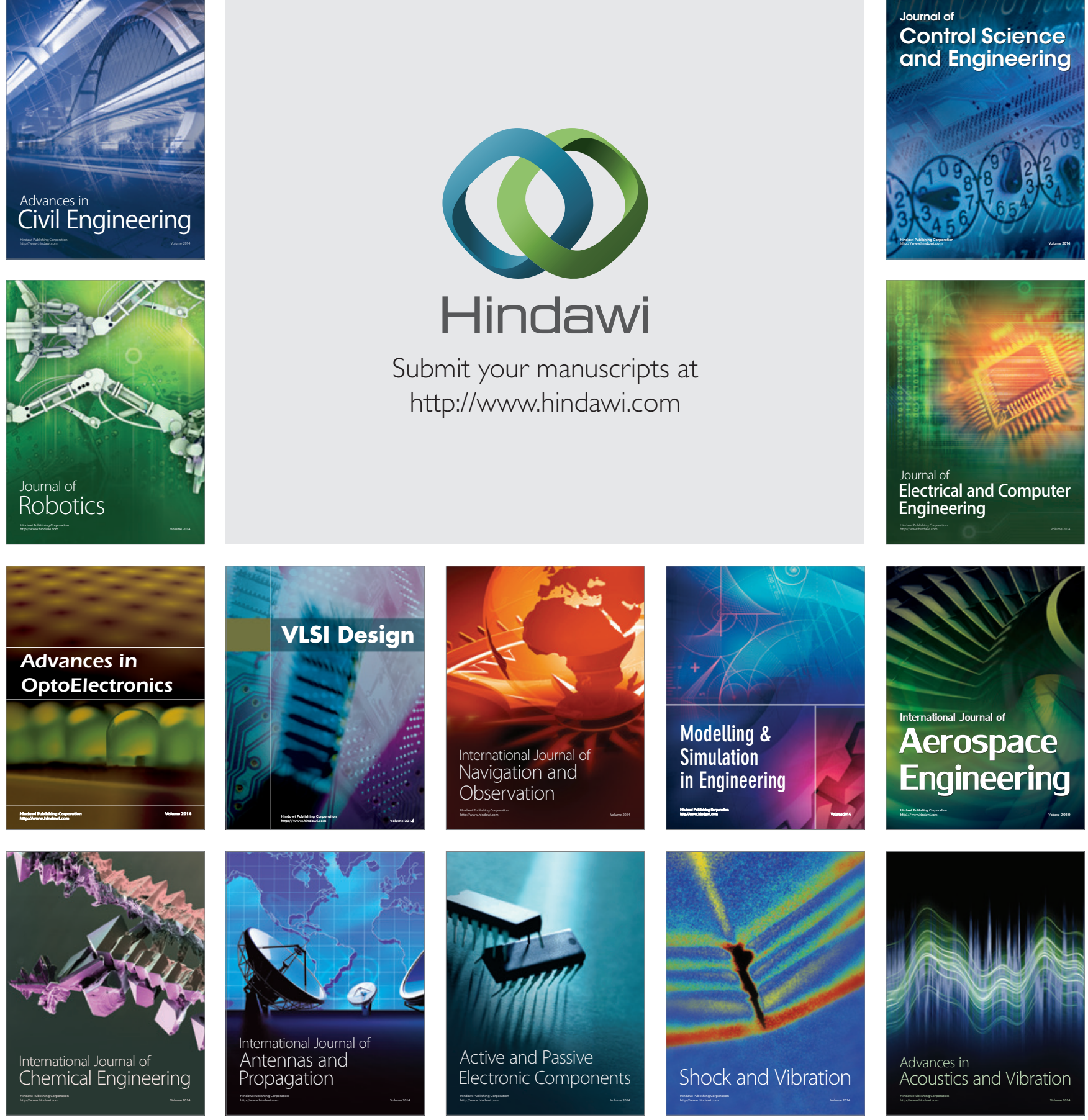